\title{
Li in NGC 6752 and the formation of globular clusters ${ }^{\star}$
}

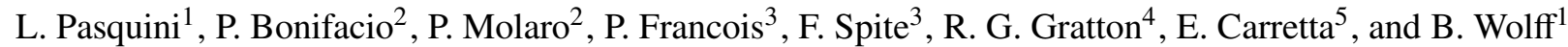 \\ ${ }^{1}$ European Southern Observatory, Garching bei München, Germany \\ e-mail: lpasquin@eso.org \\ 2 INAF - Osservatorio Astronomico di Trieste, Trieste, Italy \\ 3 DASGAL - Observatoire de Paris-Meudon, France \\ ${ }^{4}$ INAF - Osservatorio Astronomico di Padova, Padova, Italy \\ 5 INAF - Osservatorio Astronomico di Bologna, Bologna, Italy
}

Received 9 June 2005 / Accepted 22 June 2005

\begin{abstract}
Li abundances for 9 Turn-off (TO) stars of the intermediate metallicity cluster $([\mathrm{Fe} / \mathrm{H}]=-1.4)$ NGC 6752 are presented. The cluster is known to show abundance anomalies and anticorrelations observed in both evolved and main sequence stars. We find that $\mathrm{Li}$ abundance anticorrelates with $\mathrm{Na}$ (and $\mathrm{N}$ ) and correlates with $\mathrm{O}$ in these Turn-Off stars. For the first time we observe Pop II hot dwarfs systematically departing from the Spite plateau. The observed anticorrelations are in qualitative agreement with what is expected if the original gas were contaminated by Intermediate Mass AGB - processed material. However, a quantitative comparison shows that none of the existing models can reproduce all the observations at once. The very large amount of processed gas present in the cluster does not imply a "pollution", but rather that the whole protocluster cloud was enriched by a previous generation of stars. We finally note that the different abundance patterns in NGC 6397 and NGC 6752 imply different composition of the pre-enrichment ejecta for the two clusters.
\end{abstract}

Key words. stars: abundances - Galaxy: globular clusters: individual: NGC 6752

\section{Introduction}

With the advent of $8 \mathrm{~m}$ telescopes we are able to obtain high resolution and high quality spectra for stars belonging to the main sequence of globular clusters, which have allowed astronomers to derive accurate abundances for them. These abundances have set several limits to the physics of stellar atmospheres, and have shed some light on the long debated problem of the origin of chemical anomalies in globular clusters (Thevenin et al. 2001; Gratton et al. 2001, hereafter G01; James et al. 2004; Carretta et al. 2004). In this context $\mathrm{Li}$ abundance studies play a special role, given the fragility of this element which can be easily destroyed in the stellar interior. Indeed, while early Li studies in globular clusters mostly concentrated on the problems related to the primordial nature of the Li plateau (Molaro \& Pasquini 1994; Pasquini \& Molaro 1996, 1997; Delyiannis et al. 1995; Boesgaard et al. 1998), later studies have emphasized the role of Li for understanding the mixing phenomena in globular cluster stars or for constraining the mechanism responsible for the chemical pollution of the clusters (Castilho et al. 2000; Thevenin et al. 2001; Bonifacio et al. 2002; Grundahl et al. 2002). The only cluster studied in some detail as faint as the Turn-off is the nearby, metal-poor NGC 6397, which exhibits

* Based on observations collected at the ESO VLT, Paranal Observatory, Chile, Archive data of the Programme 165.L-0263. an impressively constant Li abundance among TO stars, at the same level of the field stars plateau (Bonifacio et al. 2002). Since NGC 6397 shows chemical inhomogeneity in the oxygen abundance among main sequence stars and a high nitrogen abundance, as recently reported by Pasquini et al. (2004), it is difficult to explain the plateau $\mathrm{Li}$ abundance without a fine tuning. The proposed Li production from intermediate mass (IM) AGB stars should give yields very close to the values of primordial nucleosynthesis. The presence of a significant amount of beryllium suggests that these IM-AGB stars formed very early after the big bang, and polluted the gas which was later exposed for several hundred million years to the galactic cosmic ray flux before the stars we now observe formed (see the discussion in Ventura et al. 2001, 2002; Bonifacio et al. 2002; Pasquini et al. 2004). Since the case of NGC 6397 would require a fine tuning between Li production and destruction in the IM-AGB to reproduce exactly the Li plateau level observed, it is interesting to investigate the behaviour of $\mathrm{Li}$ in other clusters to test if they carry the signature of AGB pollution. NGC 6752 has a metallicity of $[\mathrm{Fe} / \mathrm{H}]=-1.43$, and with a temperature of about $\sim 6200 \mathrm{~K}$, its TO stars belong to the Li plateau (Spite \& Spite 1982). NGC 6752 is therefore an ideal cluster for this study. The cluster is one of the prototypes of globular cluster with chemical anomalies, where the first $\mathrm{O}-\mathrm{Na}$ anticorrelation has been discovered among TO stars (G01). 
Table 1. NGC 6752 stars, their atmospheric parameters and Li abundances. Stars are ordered by increasing [Na/Fe] abundances. The first three columns report the observed photometric parameters. The G01 temperature was of $6226 \mathrm{~K}$ and the surface gravity of $\log g=4.28$ for all stars. The Li value in Col. 6 and $[\mathrm{Na} / \mathrm{Fe}]$ in Col. 7 are computed with these stellar parameters. Column 8 presents $T_{\text {eff }}$ computed according to the Alonso scale, $b-y$ colour and the reddening of $E(b-y)=0.032(\mathrm{G} 03)$. The $[\mathrm{Na} / \mathrm{Fe}]$ abundances of Col. 9 and the Li abundances in $\mathrm{Col} .10$ are computed adopting the last temperatures.

\begin{tabular}{lllllrlrrr}
\hline \hline Star & $V$ & $b-y$ & $c_{1}$ & $\begin{array}{l}\mathrm{Li} E W \\
\mathrm{~m} \AA\end{array}$ & $\begin{array}{r}A(\mathrm{Li}) \\
(\mathrm{G} 01)\end{array}$ & $\begin{array}{l}{[\mathrm{Na} / \mathrm{Fe}]} \\
(\mathrm{G} 01)\end{array}$ & $\begin{array}{r}T_{\text {eff }} \\
\mathrm{K}\end{array}$ & $\begin{array}{l}{[\mathrm{Na} / \mathrm{Fe}]} \\
\text { Alonso }\end{array}$ & $\begin{array}{r}A(\mathrm{Li}) \\
\text { Alonso }\end{array}$ \\
\hline 4428 & 17.142 & 0.366 & 0.307 & 42.8 & $2.50 \pm 0.04$ & -0.35 & 6013 & -0.28 & 2.35 \\
4383 & 17.112 & 0.360 & 0.284 & 35.9 & $2.41 \pm 0.04$ & -0.23 & 6043 & -0.17 & 2.28 \\
202316 & 17.275 & 0.354 & 0.237 & 31.7 & $2.35 \pm 0.07$ & -0.09 & 6064 & -0.04 & 2.23 \\
4341 & 17.149 & 0.345 & 0.324 & 29.4 & $2.31 \pm 0.05$ & 0.18 & 6168 & 0.20 & 2.26 \\
4458 & 17.155 & 0.343 & 0.335 & 21.2 & $2.14 \pm 0.04$ & 0.24 & 6188 & 0.25 & 2.11 \\
4661 & 17.216 & 0.342 & 0.281 & 20.9 & $2.14 \pm 0.10$ & 0.28 & 6170 & 0.30 & 2.10 \\
5048 & 17.284 & 0.353 & 0.356 & 21.4 & $2.15 \pm 0.09$ & 0.37 & 6126 & 0.40 & 2.08 \\
4907 & 17.199 & 0.354 & 0.306 & 17.9 & $2.06 \pm 0.06$ & 0.61 & 6096 & 0.65 & 1.97 \\
200613 & 17.198 & 0.377 & 0.330 & 20.5 & $2.13 \pm 0.06$ & 0.64 & 5948 & 0.73 & 1.93 \\
Average & 17.192 & & & 26.9 & 2.24 & & 6091 & & 2.15 \\
$\sigma$ & \pm 0.06 & & & \pm 8.5 & \pm 0.15 & & \pm 81 & & \pm 0.14 \\
\hline
\end{tabular}

\section{Sample selection and observations}

We selected the stars of the G01 sample. Stars numbers together with their photometric properties, atmospheric parameters, $\mathrm{Li}$ and $\mathrm{Na}$ abundances as derived in $\mathrm{G} 01$ are listed in Table 1 . These stars are in the same metallicity and temperature scale as NGC 6397 stars in G01. Although G01 showed that the temperature of the stars is compatible with a single value, since $\mathrm{Li}$ abundance is very sensitive to small differences in effective temperature, we also computed the effective temperature of each star by assuming the observed photometric values, the reddening of $E(b-y)=0.032$ (Gratton et al. 2003, G03), a metallicity of -1.43 as derived by G01 and the Alonso et al. (1996) scale. ESO archive was searched to identify UVES observations of similar stars, but no spectra of TO stars were available.

Abundances were computed with the method outlined in Bonifacio et al. (2002). Kurucz models were computed with the appropriate metallicity and temperature, and the Li abundances derived from the observed equivalent widths $(E W \mathrm{~s})$. The typical error in the $\mathrm{Li}$ abundance is of $0.05 \mathrm{dex}$. We do expect an error of up to 0.1 in $A(\mathrm{Li})^{1}$ when considering possible uncertainties of up to $100 \mathrm{~K}$ in effective temperature. However, we shall consider that the moderate reddening of the cluster $(E(B-V)=0.040)$ and the use of the same temperature scale for all the stars implies that most of this uncertainty applies to the absolute value of $\mathrm{Li}$ abundance, but much less to the results concerning the dispersion of $\mathrm{Li}$ abundance in the cluster. The latter should be dominated by the photometric error in the $b-y$ colour, which should not exceed 0.02 . This translates in an uncertainty of $\approx 60 \mathrm{~K}$ in the $T_{\text {eff }}$ or $0.05 \mathrm{dex}$ in $A(\mathrm{Li})$.

\footnotetext{
${ }^{1}$ For chemical abundances we use the notation $A(\mathrm{X})=$ $\log (\mathrm{X} / \mathrm{H})+12$.
}

\section{Discussion}

The equivalent width measurements of Table 1 indicate at first glance a variability of Li. We know, however, that to establish the real $\mathrm{Li}$ variations in a cluster requires a proper analysis of the errors and of the additional, possible hidden biases introduced by the analysis method (see e.g. Bonifacio 2002). Since the stars are consistent with a single effective temperature we start the analysis by considering the equivalent widths only. The S/N ratio of the observations varies between 34 and 67 (per pixel) and the errors estimated in the equivalent widths range between $1.8 \mathrm{~m} \AA$ for the best exposed spectra to $4 \mathrm{~m} \AA$ for those with lower $\mathrm{S} / \mathrm{N}$. (The relative $\mathrm{S} / \mathrm{N}$ ratio among the stars can be deduced by the errors in the $\mathrm{Li}$ abundances given in Col. 6). The difference in Li equivalent width among different stars is between 5 to 10 times larger than the typical measurement error for any object. The average of the equivalent widths is of $26.9 \mathrm{~m} \AA$, with a $\sigma$ of $8.5 \mathrm{~m} \AA$. The dispersion is $2-5$ times larger than the measurement errors on the single spectra. In Fig. 1 the Li equivalent widths vs. Na abundance are plotted. The figure shows a clear anticorrelation between $\mathrm{Li}$ and $\mathrm{Na}$, and the Kendall's $\tau$ test provides an anticorrelation probability of $99.78 \%$.

To exclude that $\mathrm{Li}$ variations could be an artifact produced by possible temperature differences among the stars, we recomputed the $\mathrm{Li}$ abundances adopting the Alonso et al. $T_{\text {eff }}$ values given in Col. 8 of Table 1. The resulting Li abundances show a lower Li mean level (this is not surprising, being the photometric scale $135 \mathrm{~K}$ lower) and a Li scatter slightly lower but comparable with what obtained with the unique temperature hypothesis. This confirms the presence of the $\mathrm{Li}-\mathrm{Na}$ anticorrelation.

With this temperature scale the $[\mathrm{Na} / \mathrm{Fe}]$ abundances will also change. But, since $[\mathrm{Na} / \mathrm{Fe}]$ increases by decreasing the temperature $([\mathrm{Na} / \mathrm{Fe}]$ increases by 0.034 dex for a difference in temperature of $-100 \mathrm{~K}$, see G01) while $\mathrm{Li}$ abundance is 


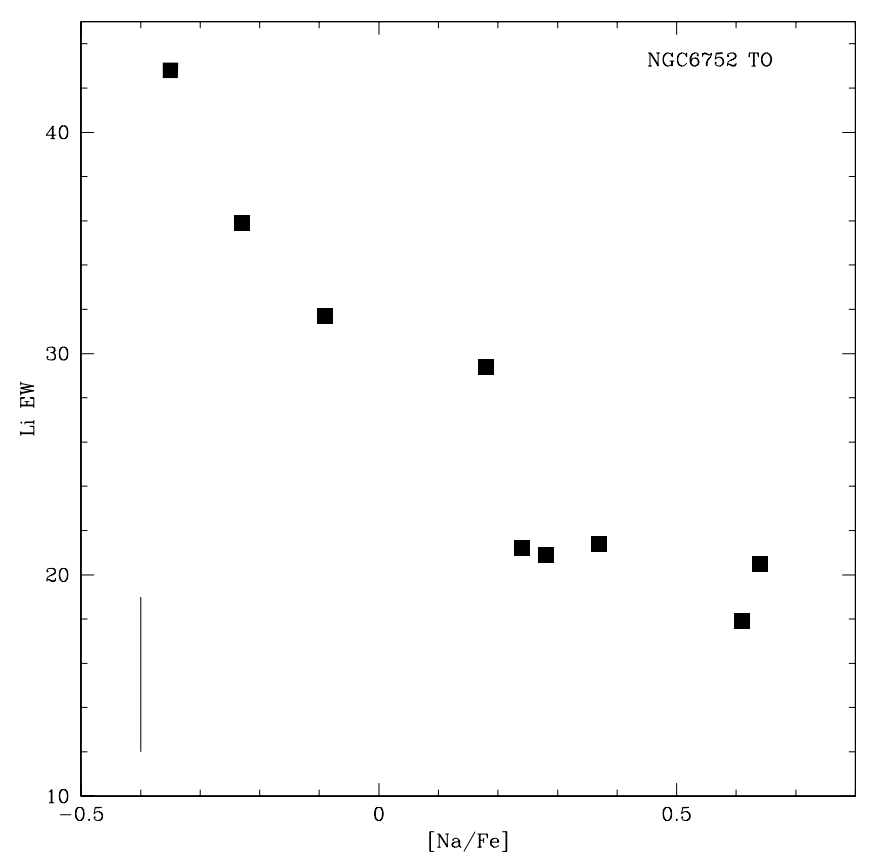

Fig. 1. Li equivalent widths vs. $[\mathrm{Na} / \mathrm{Fe}]$ for the NGC 6752 stars. The typical measurement error bar is given in the left bottom corner.

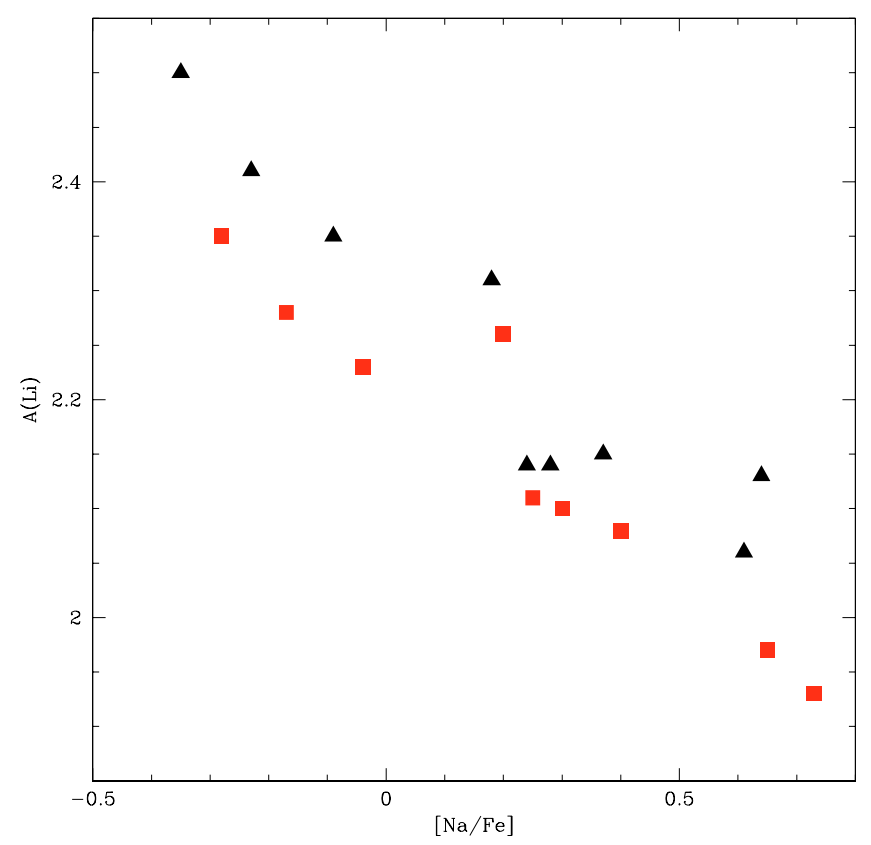

Fig. 2. $\mathrm{Li}$ abundances vs. $[\mathrm{Na} / \mathrm{Fe}]$ for the sample stars. For the sake of completeness, abundance values for both temperature scales are plotted: filled triangles G01 scale; filled (red) squares: Alonso scale.

decreasing, the Li-Na relationship remains substantially unchanged, as can be seen in Fig. 2 and by directly comparing the values tabulated in Table $1:$ the $[\mathrm{Na} / \mathrm{Fe}]$ range spanned by the stars is about one order of magnitude, irrespective of the $T_{\text {eff }}$ scale used.

We therefore are confident we found evidence for the first time of $\mathrm{Li}-\mathrm{Na}$ anticorrelation in a group of GC stars with characteristics (metallicity, temperature and gravity) close to those of the Spite plateau.

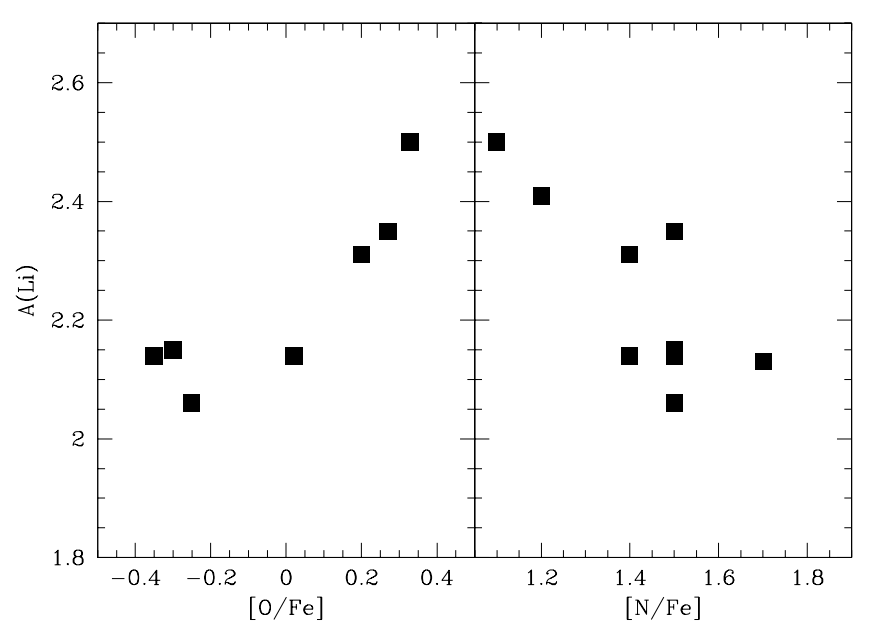

Fig. 3. $\mathrm{Li}$ abundances vs. $[\mathrm{O} / \mathrm{Fe}]$ and vs. $[\mathrm{N} / \mathrm{Fe}]$ for the sample stars. $[\mathrm{O} / \mathrm{Fe}]$ and $[\mathrm{N} / \mathrm{Fe}]$ values are from Table 1 of Carretta et al. (2005), who used the G01 temperature scale.

There is no general consensus about the metallicity at which the plateau ends in the field halo dwarfs. Bonifacio \& Molaro (1997) defined this edge to occur around $[\mathrm{Fe} / \mathrm{H}]=-1.5$, where the first signs of stellar Li depletion start to appear. NGC 6752 with $[\mathrm{Fe} / \mathrm{H}]=-1.4$ is near this edge, being in fact slightly more metal-rich. The metal enhancement is however so small (and also dependent on the zero point adopted) to make the belonging of these TO stars to the plateau unquestionable.

We interpret the Li-Na anticorrelation as evidence that the gas forming NGC 6752 has been contaminated by a previous population which is responsible for the chemical inhomogeneities.

We must analyze the extent to which our targets have been polluted by the processed material. This aspect, in turn, will also provide us with fresh information about the details of the cluster formation.

\subsection{Implications on cluster chemical anomalies and cluster formation}

Figure 2 shows the behaviour of $\mathrm{Li}$ abundances vs. Na abundances in our stars. In NGC $6752 \mathrm{Na}$ anticorrelates with $\mathrm{O}$ (G01), and correlates with N (Carretta et al. 2005); in Fig. 3 the behaviour of $\mathrm{Li}$ with $[\mathrm{O} / \mathrm{Fe}]$ and $[\mathrm{N} / \mathrm{Fe}]$ is shown, confirming the correlation with $[\mathrm{O} / \mathrm{Fe}]$, while the same anticorrelation observed for $\mathrm{Na}$ is recorded for $\mathrm{N}$. The most immediate explanation is that the Na-poor stars, which are also $\mathrm{O}$ and $\mathrm{Li} \mathrm{rich,}$ have a composition close to the "pristine" one, while the $\mathrm{Na}$ (and $\mathrm{N}$ ) rich stars are progressively contaminated.

An important point to recall is that the CNO cycle, which makes the $\mathrm{N}$ overabundance and $\mathrm{O}$ underabundance, and the Ne-Na cycle which produces the $\mathrm{Na}$ overabundance occur at very high temperatures, 20-30 times higher than the $\sim 2.5$ Million $\mathrm{K}$ at which $\mathrm{Li}$ is destroyed. It is thus expected that in the places where these cycles occur, no Li is left. If "pristine" and "processed" material are mixed, then $\mathrm{Li}, \mathrm{Na}$ and $\mathrm{N}$ are expected to show some anticorrelation, and $\mathrm{Li}$ and $\mathrm{O}$ some correlation. 
In Figs. 1 and 2 there are two additional relevant aspects to be considered. A first aspect concerns the stars with the higher Li. These stars show the lowest $\mathrm{Na}$ and highest $\mathrm{O}$ and most likely they are very little polluted by processed material. If we take the $\mathrm{Li}$ abundance of these stars on the G01 temperature scale at face values, their $A(\mathrm{Li})$ is about 2.45 , or $0.1 \mathrm{dex}$ higher than the plateau level. We note that these values are also found in NGC 6397 stars when adopting the same temperature scale (Bonifacio et al. 2002, their Col. 7 in Table 2). We interpret therefore this higher $\mathrm{Li}$ abundance as entirely due to the use of the G01 temperature scale, which is hotter than the Alonso and the Bonifacio et al. (2002) temperature scales.

A second aspect refers to the most Li-poor stars: it is worth noticing that also in the most $\mathrm{Li}$-poor stars the $\mathrm{Li}$ line is always detected, although at an abundance level of $A(\mathrm{Li}) \sim 2$, or 2-3 times lower than in the stars with the highest Li content. The fact that some $\mathrm{Li}$ is preserved even in the most Na-rich stars confirms that the observed chemical anomalies have not been produced by the star itself, but rather that the gas was processed previously somewhere else. This was shown by G01, because TO stars should not reach temperatures so high to ignite the Na cycle. However, this behaviour is different from what observed in the metal poor cluster NGC 6397, where $A(\mathrm{Li})$ is constant.

The contamination can be obtained in different ways either through Bondi accretion or through a process involving the whole protocluster cloud. We favour the latter because if the chemical anomalies were limited to the external accreted layers of the star, they should be later washed out when the stars undergo the first dredge up, (as happens for Li, cf. Grundahl et al. 2002). This is not the case, since these anomalies are observed all along the RGB (see e.g. Carretta et al. 2005). The fact that $\mathrm{Li}$ is observed even in the most "contaminated" stars implies, then, that some Li must have been created by the previous generation of (contaminant) stars.

The second possibility however implies such a huge contamination of the protocluster cloud that probably "contamination" is not the most appropriate term anymore.

The anomalous abundances suggest a precise composition of the contaminating gas. The maximum difference observed in the $\mathrm{Na}$ abundance is almost one order of magnitude, the one in the Oxygen abundance is of about $0.8 \mathrm{dex}$, in the Li abundance is only of about a factor 2.5. At the same time the other heavier elements remain unchanged, and in particular the accreted material was not enriched in s-process elements (James et al. 2004). This shows that the most polluted stars have accreted at least $90 \%$ of their gas which was $\mathrm{Na}$ rich, with a $\mathrm{Li}$ content lower but close to primordial and a negligible content of Oxygen. If a large fraction of the stars' mass is indeed made out by this processed material, it is likely that this is just the signature of a group of stars in a limited mass range. We can draw a scenario where the elements created by supernovae are well mixed in the protocluster, while the products of stellar winds, with lower velocity, would be more inhomogeneous.

The ejecta of the previous generation of stars had an upper limit content of $A(\mathrm{Li}) \sim 2.0 ; \mathrm{A}(\mathrm{Na})$ of at least $\sim 5.4 ; \mathrm{A}(\mathrm{O})$ of less than $\sim 7.0$ and $A(\mathrm{~N}) \sim 7.9$. The general behaviour is qualitatively consistent with the models by Ventura et al. $(2001,2002)$ who predicted the Li-O correlation from an intermediate-mass AGB contamination and that Li should not be destroyed completely.

In more quantitative terms, there is a rather good agreement with the models published by Ventura et al. (2002) for very low metallicity IM-AGB. Ventura et al predict, for a $Z=0.0006$ initial composition, Li abundance of the order of $A(\mathrm{Li}) \sim 1.5-2$, oxygen abundance of $\sim 6.5-7.4$ and $N$ abundance of $\sim 6.9-8.3$. Nitrogen abundance in NGC 6752 TO stars is enhanced (Carretta et al. 2005) showing clear evidence for CNO processing. According to the models, the low oxygen abundance provides a clear indication that the generation of stars producing the chemical inhomogeneities in NGC 6752 could only originate from 4-5 $M_{\odot}$ metal poor (with $Z<$ 0.0006) AGB stars.

The relatively high $\mathrm{Li}$, on the other hand, is predicted to be produced only by fairly low mass ( 3 solar masses) and relatively metal rich progenitors. Although a full, detailed modelling might change these results, our preliminary conclusions are that the observed oxygen and Li abundances seem incompatible with progenitors of one type. We note that other works found that the oxygen-Na anticorrelation cannot be quantitatively explained by the present IM-AGB models (Denissenkov \& Herwig 2003; Palacios et al. 2005; Ventura \& D'Antona 2005). Uncertainties in fundamental aspects of AGB evolution such as mass loss rate and treatment of convection at present seem to hamper the generation of realistic predictions for low metallicity AGB stars, and we might be at the stage where observations such as those presented here will serve to constrain evolutionary models rather than the opposite.

Another important aspect is to understand the difference between the Li behaviour in NGC 6397 and in NGC 6752 if the IM-AGB scheme were acting in both clusters. A corollary implication would be that the ejecta of the contaminants of the two clusters had different chemical compositions. Following the same argument as above and taking the $\mathrm{O}$ and $\mathrm{N}$ data from Pasquini et al. (2004), for NGC 6397 we expect ejecta which were more rich in $\mathrm{Li}$ (about 2 times, or $A(\mathrm{Li}) \sim 2.3$ ), with an O-poor content of $A(\mathrm{O})$ of less than $\sim 6.7$, while they had about $A(\mathrm{~N}) \sim 7.3$. As far $\mathrm{Na}$ is concerned, the value measured by $\mathrm{G} 01$ in the TO stars is at the level of $A(\mathrm{Na}) \sim 4.5$, constant among all stars, but the analysis of the subgiants by Carretta et al. (2005) shows clear variations with values of $A(\mathrm{Na})$ up to $\sim 4.8$.

We finally comment that in order to explain the chemical variations observed in the AGB context, huge pollution is required. The two stars $\mathrm{n} 4907$ and $\mathrm{n} 200613$ should have been formed by more than $\sim 90 \%$ of IM-AGB processed material. If our sample of stars is indicative of the cluster population, it would imply that a large fraction (say about a half) of the gas which formed the stars we now observe was indeed processed by the previous IM-AGB stars population. The actual cluster mass is about $2 \times 10^{5}$ solar masses, therefore at least $10^{5}$ solar masses were processed by IM-AGB stars, leading to a minimum of $\sim 3 \times 10^{4}$ IM-AGB stars to produce the observed anomalies. Since there is no hint of the presence of low-mass stars belonging to this first generation, this implies a flat-topped IMF. A parallel effect would be He enhancement produced by this IM-AGB processing, which was analyzed by 
Ventura et al. (2002) and by D'Antona et al. (2003). In addition, a considerable number of remnants should be present in the cluster. These white dwarfs might be, however, not easily detectable: they would be likely in the faint tail of the luminosity function and, in addition, they might have been segregated during the complex dynamical history of the cluster.

Even if at present AGB stars remain the most promising candidates, the problems encountered in explaining all the observed features lead some groups to look for alternative scenarios to explain the observed abundance patterns: Yong et al. (2005) suggested the presence of a new process producing simultaneously light and s elements in globular clusters; Piotto et al. (2005) invoked the possible presence of low mass $\mathrm{SNe}$ to explain the He-rich main sequence of $\Omega \mathrm{Cen}$, and $\mathrm{SNe}$ with extensive fall-back were invoked in various flavours to explain the abundance patterns of the most metal poor stars (Umeda \& Nomoto 2003; Limongi et al. 2003). Massive stars able to eject light elements, while retaining the heavy elements locked in the SN remnant are, in principle, attractive candidates. However, when analysing possible scenarios, we encounter several problems: it is difficult, for instance, to produce the very low oxygen observed, to locate a process of Li production, and, given the enormous mass of processed material required, a very peculiar IMF must be postulated.

Quantitative element analysis, such as that presented here, provide the experimental framework for solving this interesting puzzle.

Acknowledgements. We thank Gabriella Schiulaz for a careful reading of the manuscript.

\section{References}

Alonso, A., Arribas, R., \& Martinez-Roger, C. 1996, A\&AS, 117, 227 Boesgaard, A. M., Deliyannis, C. P., Stephens, A., \& King, J. R. 1998, ApJ, 493, 206

Bonifacio, P. 2002, A\&A, 395, 915
Bonifacio, P., \& Molaro, P., 1997, MNRAS, 285, 847

Bonifacio, P., Pasquini, L., Spite, F., et al. 2002, A\&A, 390, 91

Carretta, E., Bragaglia, A., Gratton, R. G., Bonifacio, P., \& Pasquini, L. 2004, A\&A, 416, 925

Carretta, E., Gratton, R.G., Bragaglia, A., Lucatello, S., \& Bonifacio, P. 2005, A\&A, 433, 597

Castilho, B. V., Pasquini, L., Allen, D. M., Barbuy, B., \& Molaro, P. 2000, A\&A, 361, 92

D' Antona, F., et al. 2003, in New Horizons in Globular Cluster Astronomy, ed. G. Piotto, G. Meylan, S. G. Djorgovski, \& M. Riello, 296, ASP Conf. Proc., 293

Denissenkov, P. A., \& Herwig, F. 2003, ApJ, 590, L99

Deliyannis, C., Boesgaard, A. M., \& King, J. R. 1995, ApJ, 452, L13

Gratton, R. G., Bonifacio, P., Bragaglia, A., et al. 2001, A\&A, 369, 87 (G01)

Gratton, R. G., Bragaglia, A., Carretta, E., et al. 2003, A\&A, 408, 529 (G03)

Grundahl, N., Briley, M., Nissen, P. E., \& Feltzing, S., et al. 2002, A\&A, 385, L14

James, G., Francois, P., Bonifacio, P., et al. 2004, A\&A, 414, 1071

Limongi, M., Chieffi, A., \& Bonifacio, P. 2003, ApJ, 594, L123

Molaro, P., \& Pasquini, L. 1994, A\&A, 281, L77

Palacios, A., Charbonnel, C., Talon, S., \& Siess, L. 2005, in Chemical abundances and mixing in stars in the Milky Way and its satellites, ed. S. Randich, \& L. Pasquini, in press

Pasquini, L., \& Molaro, P. 1996, A\&A, 307, 761

Pasquini, L., \& Molaro, P. 1997, A\&A, 322, 109

Pasquini, L., Bonifacio, P., Randich, S., Galli, D., \& Gratton, R. G. 2004, A\&A, 426, 651

Piotto, G., Villanova, S., Bendin, L. R., et al. 2005, ApJ, 621, 777

Spite, F., \& Spite, M. 1982, A\&A, 115, 357

Thévenin, F., Charbonnel, C., de Freitas Pacheco, J. A., et al. 2001, A\&A, 373, 905

Umeda, H., \& Nomoto, K. 2003, Nature, 422, 871

Yong, D., Grundahl, F., Nissen, P. E., Jensen, H. R., \& Lambert, D. L. 2005 [arXiv: astro-ph/0504283]

Ventura, P., Mazzittelli, I., D’ Antona, F., \& Gratton, R. G. 2001, ApJ, 550, L65

Ventura, P., D’Antona, F., \& Mazzitelli, I. 2002, A\&A, 393, 215

Ventura, P., \& D’ Antona, F. 2005, A\&A, 431, 279 